\title{
An Augmented Web Space for Digital Cities *
}

\author{
Kaoru Hiramatsu \\ NTT Communication Science Laboratories \\ 2-4, Hikaridai, Seika-cho, Soraku-gun \\ Kyoto, 619-0237 Japan \\ hiramatu@cslab.kecl.ntt.co.jp
}

\author{
Toru Ishida \\ Department of Social Infomatics, Kyoto University \\ Yoshidahonmachi, Sakyo-ku \\ Kyoto, 600-8501 Japan \\ ishida@i.kyoto-u.ac.jp
}

\begin{abstract}
We propose an augmented Web space and its query language to support geographical querying and sequential plan creation utilizing a digital city that is a city-based information space on the Internet. The augmented Web space involves a new approach to integrate the World Wide Web $(W W W)$ and a geographic information system (GIS). The augmented Web space consists of home pages (HP), hyperlinks, and generic links that represent geographical relations between HPs. The generic links are created dynamically using geographical evaluation functions included in a user's search query each time one is issued. A query also includes a path expression showing how to navigate the HPs, hyperlinks, and generic links. Since the path expression is an extended regular expression, we can describe an arbitrary sequence of users' search actions for navigating the augmented Web space. We have applied the proposed augmented Web space to Digital City Kyoto, a city information service system that is accessed through a $3 D$ walk-through implementation and a map-based interface. Each time a user's query is issued through the $3 D$ and $2 D$ interfaces, Digital City Kyoto creates an augmented Web space, and navigates the Web information space based on the path expression in the query.
\end{abstract}

\section{Introduction}

City information is currently being accumulated as online digital contents for a GIS and the WWW[5]. This online city data is enabling us to have a highly integrated information system to support everyday life in a metropolitan area. At the same time, as mobile phones are becoming

\footnotetext{
*(C) 2001 IEEE. Personal use of this material is permitted. However, permission to reprint/republish this material for advertising or promotional purposes or for creating new collective works for resale or redistribution to servers or lists, or to reuse any copyrighted component of this work in other works must be obtained from the IEEE.
}

more technologically sophisticated, accessibility to the Internet in urban life is becoming easier and more popular. It will not be very long before mobile phones turn into information devices that allow users to have conversations with interface agents. Let us consider the following example.

'Today is Saturday. John has a dinner appointment with Mary. However, he first needs to finish writing a paper at his university and mail it from a post office. He asks an agent on his mobile phone, "would you find the post office that is nearest to my university and is also open on Saturday?" While the agent is searching, John calls Mary. She says she cannot wait because she is hungry and will meet him at the restaurant. John now asks the agent, "Please check a restaurant information site. Are there any good Italian restaurants within one kilometer of the post office? I would prefer one with a large wine selection. ",

There are many HPs on the Internet. These HPs are linked together by hyperlinks. A GIS stores urban building information with geographical attributes. If we can use not only the hyperlinks in a Web space but also the links representing geographical relations, we can put inquiries such as the one in the above scenario into practice.

For example, we first parse the above scenario for querying purposes. In the first inquiry about searching for " a post office that is nearest from my university and is open on Saturday," we extract the university address from a Web page of his university. The extracted address is the key to the next search for the nearest post office in the GIS. The final target is the HP of the post office and we check whether it includes the string "open on Saturday." The second query is "Please check a restaurant information site. Are there any good Italian restaurants within one kilometer of the post office? I would prefer one with a large wine selection." The starting point of the second search is the restaurant information site. The search selects restaurant HPs that contain a long wine list. We then extract the restaurant addresses from their HPs, and search for the restaurants in the GIS that are within one kilometer of the post office.

As demonstrated above, the ability to search HPs with 
geographical attributes is quite useful. In this paper, we propose the creation of an augmented Web space that is an extension of a Web space that integrates geographical relations between HPs ${ }^{1}$.

In the augmented Web space, all Web information is regarded as a semi-structured database [2]. An inquiry into the augmented Web space is expressed as a search path describing an ordered sequence of HPs, hyperlinks, and generic links.

In this paper, we attempt to define a generic link and a path expression, which are key components of the augmented Web space. We also propose an application program interface to apply the augmented Web space to Digital City Kyoto. Digital City Kyoto has about 2600 links to HPs accessible by the public: restaurants, schools, shops, temples, bus stops, and so on. Each link has precise geographical attributes expressed as longitude and latitude. The examples in this paper demonstrate how to carry out a search scenario in the augmented Web space.

\section{Related Work}

\subsection{Semi-structured Database}

Query languages for Web space search is a hot topic in the database research community. W3QL [6][7] and WebSQL [9][10] are well known research results in this area. Both languages are for semi-structured databases including the WWW, where HPs are described with HTML tags hierarchically, and linked with each other[2]. W3QL uses a Web space model that regards the Web space as a globally distributed hypertext database. WebSQL introduces a relational model and a graph structure that integrate nodes and links for WWW search.

Both W3QL and WebSQL are query languages extended from SQL. Each search request is expressed as a sequence of conditions of HP attributes and link attributes. Since it is hard to estimate the position of the target data on the WWW, the search sequence of the query in either language is described using a regular path expression.

\subsection{Geographical Relational Openators}

Geographical relational operators have been standardized by the Open GIS Consortium, Inc. [12]. The consortium has proposed geographical models of $2 \mathrm{D}$ or $3 \mathrm{D}$ objects, relational operators among objects, and SQL for database operations. The objects include classified attributes such as positions, shapes, addresses, and owners. The relational operators enable us to evaluate object relations such as Touches, Within, Crosses, and Overlaps.

\footnotetext{
${ }^{1}$ We are not concerned here with agent technology and mobile equipment. Discussions on such matters will be reported in another article.
}

\subsection{Web-based GIS}

Many pilot systems have been reported in the WWW research community. This has enabled us to browse GIS information from the WWW. Potmesil [13] has developed two types of geographical browsers: a 2D map browser capable of the continuous scroll and zoom of an arbitrarily large sheet and a 3D flight-simulator browser capable of continuous flight around the Earth. Gis2web [11] is a system that bridges a GIS and the WWW and enables us to access GIS information and WWW information seamlessly.

\section{Augmented Web Space}

This section describes the basic architecture of an augmented Web space and its two key components: a generic link and a path expression.

\subsection{Basic Idea}

We propose an augmented Web space that is obtained by dynamically extending a Web space with generic links, which represent geographical relations between HPs in a physical city (Fig.1). Though various kinds of links can be defined as a generic link, this paper focuses on generic links based on geographical information. This extension is triggered by users' queries, which contain geographical evaluation functions. The generic links are created dynamically and therefore do not explicitly appear on HPs.

We have designed a query language for the above augmented Web space, based on the query languages available for Web space search. Here, because geographical relations on HPs are basically independent of the WWW hyperlink network, and are not defined as HTML tags, we use geographical operators defined by Open GIS [12]. Figure 3 shows an example of a generic link that is obtained by applying GIS geographical operators to HPs.

To search the augmented Web space, we use extended regular expressions in order to handle the semi-structured Web space. These path expressions specify sequences of HPs and hyper/generic links. The rest of this section explains two key components of the augmented Web space: a generic link and a path expression.

\subsection{Generic Link}

We define the two types of links in the augmented Web space: one is a hyperlink (Fig.2) and the other is a generic link (Fig.3). The rectangles in Fig.2 indicate HPs and the line between HPs represent links.

The hyperlink, one of the basic features for normal Web browsing, is a directed link between HPs that indicates an anchor object of HTML. Each search path in the augmented 


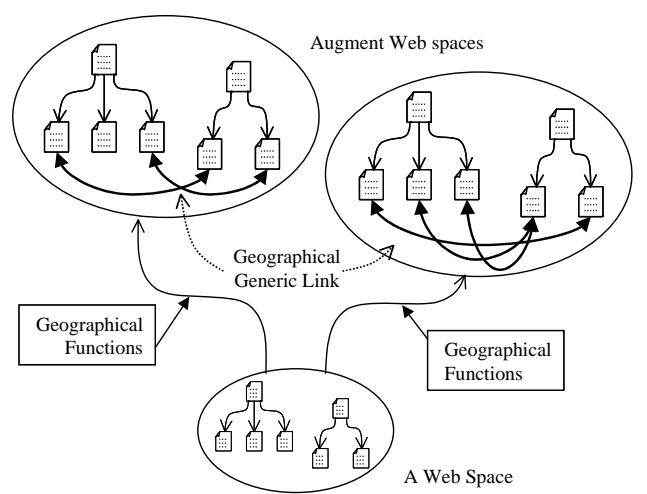

Figure 1. Examples of augmented Web spaces.

Web space is a sequence of conditions of HP attributes such as text segments and images as well as conditions of the host, directory, and file names indicated by the URL of the hyperlink.

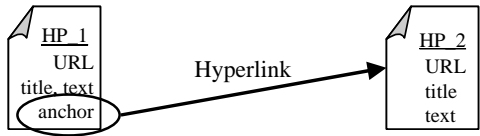

Figure 2. Hyperink.

The generic link is a link dynamically created based on inter-HP relations, which is not explicitly described on the HPs. Here, we are especially concerned with geographical generic links. Such links can express geographical relations in an actual city: examples include "a department store HP_a that is next to another department store HP_b," "bus stops HP_d within 100 meters of the city office HP_c," and so on.

In previous works, related ideas for inexplicitly described links were proposed for TextLink-III [15] and TextLink/Gem [14]. The authors of these works introduced a query-pair link that is a pair consisting of a source query and a target query for a hypertext document. Lexical chaining [4] is utilized for automatic hypertext generation that is based on information retrieval methods. Backlinks [3] are also automatically generated from existing Web information. Unlike these works, our generic link is not another way to represent a static hyperlink, but is created dynamically when triggered by users' search actions.

The generic link shown in Fig. 3 connects a starting HP with the target HP by using the geographical relation of the HPs' attributes. The creation takes three steps: mapping from the starting HP to a GIS object, evaluating the ge-

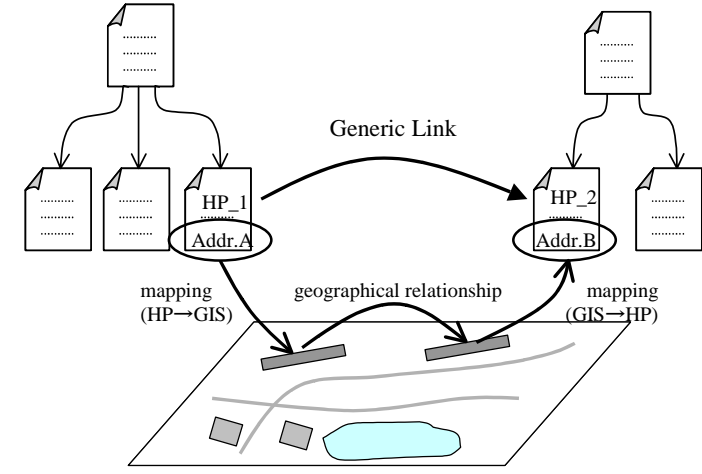

Figure 3. An example of a generic link according to geographical relations.

ographical relations between GIS objects, and remapping from a GIS object to the target HP. First, in the mapping from the starting HP to a GIS object, geographical attributes are retrieved from the starting HP such as an address, a shop name, and a page title. These attributes are projected onto the GIS object. Next, geographical relational operators such as Touches and Distance are applied to GIS objects to select all of the objects that match the geographical condition. Finally, a generic link is composed by searching for HPs that have common attributes of the GIS objects, e.g., addresses, shop names, and their owners.

In the actual augmented Web space, however, the mapping from an HP to GIS objects and the mapping from GIS objects to HPs are not always completed. This is caused by the incompleteness of information sources or variations of vocabularies in HP attribute descriptions ${ }^{2}$.

There is also apprehension about the invasion of individual privacy if precise position data can be retrieved from an HP. To avoid this problem, our database consists of triples each of which is an HP URL, its precise coordinates, and the HP owner's permission. All of the HPs in the database are allowed to be retrieved from a Web space only if permission is obtained from the HPs' owners.

\subsection{Path Expression}

In the augmented Web space, a search path expression defines a sequence of conditions about HP attributes and hyper/generic links. The path expression in this paper is an extension of the pattern graph of W3QL [6][7].

In our path expression, the path from a starting HP to a target HP is represented by a set of HPs and the links be-

\footnotetext{
${ }^{2}$ These problems have been taken up in various research areas such as natural language processing, information retrieval, and the integration of heterogeneous databases. However, we are not concerned with these incomplete cases in this paper.
} 
tween the HPs. For example, Fig.2 is represented as HP_1, Hyperlink, HP_2 and Fig.3 is represented as HP_1, GenericLink, HP_2. Path expressions containing more than two HPs are shown in Fig.4. Figure 4(a) is a simple extension of the pattern in Fig.2. The path is expressed as HP_1, Link_1, HP_2, Link_2, HP_3.

In Fig. 4(b), the path includes a self-loop for handling arbitrary Web structures: HP_1, Link_1, (HP_2,Link_2), Link_3, HP_3, where (HP_2,Link_2) represents the self-loop of HP_2 and Link_2. This expression represents a search path from a starting HP to a target HP including finite links in arbitrary Web structures. The self-loop shows that the evaluation of (HP 2,Link_2) continues until the condition of Link_3 is satisfied. In the example of searching for restaurants in Section 1, a path with a self-loop would enable us to navigate all of the links from the top page to the target page of an Italian restaurant with a long wine list.

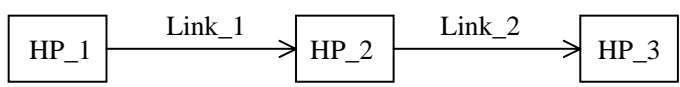

(a) Search path (cascade type)

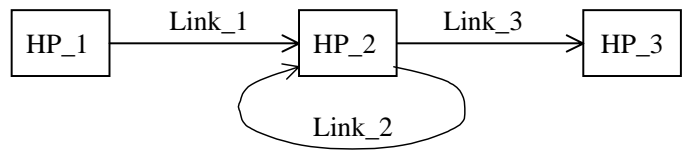

(b) Search path with self loop

\section{Figure 4. Path patterns by search path expres- sion.}

\section{Query Language}

In this section, we define the syntax of a query language and enumerate a couple of search path expressions by using illustrations. The query language is based on the generic links and the search path expressions of the augmented Web space.

\subsection{Syntax}

The query language of the augmented Web space is designed mainly based on W3QL [6][7]. We modified the part for handling and evaluating attributes of HPs and integrated them with generic links created dynamically using geographical evaluation functions included in a user's search query each time one is issued. The search expression is described in the form of SELECT-FROM-WHERE as a standard SQL query. The grammar of this query language is shown in Fig.5.

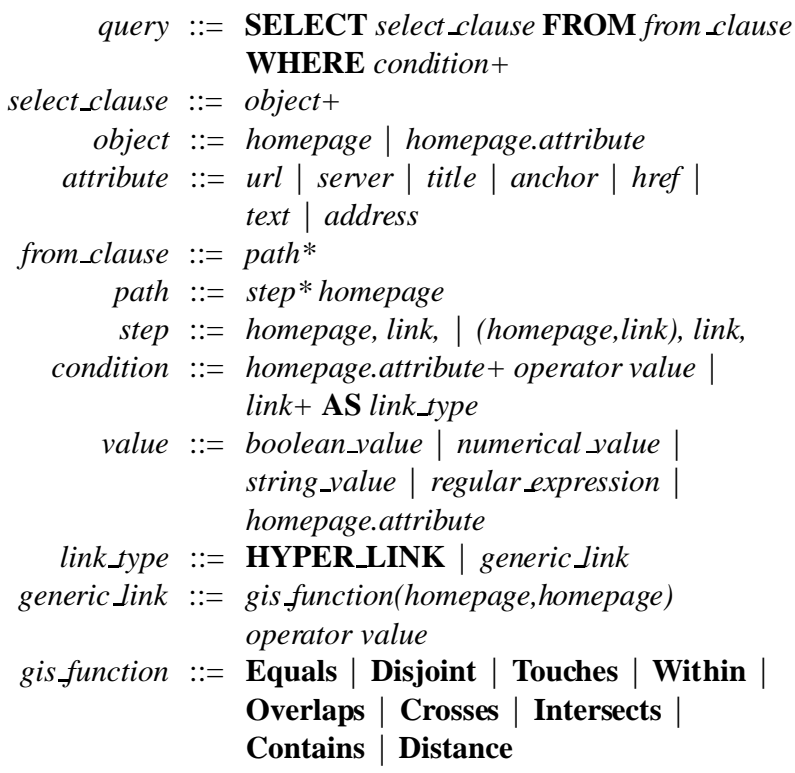

Figure 5. Grammar of query language.

In this figure, words in all capital letters and words beginning with a capital letter denote grammar terminals and lower case letters denote nonterminals. A symbol * means zero or more repetitions of a construct and + means one or more repetitions. A value expresses a boolean value, a numerical value, a string, a regular expression of a string, or an HP attribute in a search path expression. An operator is a Perl binary operator such as a sign of equality or inequality for a numerical value or a binding operator for strings.

\subsection{Semantics}

A nonterminal select_clause is an enumeration of an HP homepage and HP attribute homepage.attribute as a search result. A from_clause denotes a search path expression path* from a starting HP to a target HP as stated in Section 3.3; it unfolds according to the condition. An expression with homepage and link in parentheses denotes the self-loop of a part of a search path that is adapted to semi-structured WWW information.

A nonterminal condition following WHERE denotes the condition of an HP, HP attributes, the type of link between HPs, or a characteristic of a link on a search path. The condition is evaluated in terms of its order of appearance on the search path. If two or more conditions are enumerated on the search path, they will be evaluated as product conditions.

Links are classified into one of two types according to the link type following the termination of AS. One is a hyperlink between HPs and the other is a generic link. The hyperlink denoted as HYPER_LINK is organized if the former HP includes an anchor tag to the latter HP. There are 
also different kinds of hyperlinks extended by search engines and form HPs, but here we limit the discussion to the simple hyperlinks and the generic links. The generic link is established if a specified geographical relation exists between the former HP and the latter HP.

The geographical relation is created by using GIS functions. These functions estimate the numerical value of the relationship between the actual objects mapped from HPs or determine whether they have the specified relationship or not.

\section{Table 1. Examples of GIS functions for creat- ing generic links.}

\begin{tabular}{|l|l|}
\hline function name & return value \\
\hline Equals & boolean value \\
Disjoint & boolean value \\
Touches & boolean value \\
Within & boolean value \\
Overlaps & boolean value \\
Crosses & boolean value \\
Intersects & boolean value \\
Contains & boolean value \\
Distance & continuous value \\
\hline
\end{tabular}

\subsection{Examples}

\section{(1) Search example based on generic links}

The expression in Fig. 6 denotes a search path from the starting HP of a building to the target HPs of buildings that exist within 100 meters of the starting point.

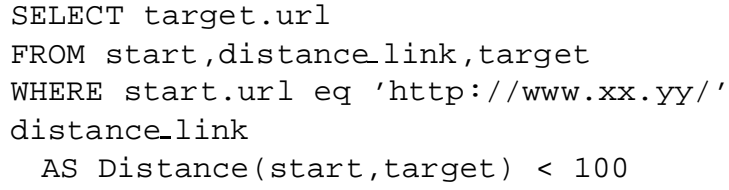

\section{Figure 6. A query examples based on generic} links.

The URL of the starting HP is given on line 3. The search path expression on line 2 is composed of the starting HP start, the target HP target, and the link distance_link. The link distance_link on line 4 is defined as a generic link that is created by evaluating the distance function Distance between the objects mapped from the HPs. The select-clause on line 1 specifies the result format when outputting target.url.

In this search example, no requirements about the target are specified so that all HPs, from which GIS objects are mapped, within 100 meters of the building of the starting HP are listed.

\section{(2) Search path expression with self-loop}

The query example in Fig. 7 includes the self-loop shown in Fig.4(b).

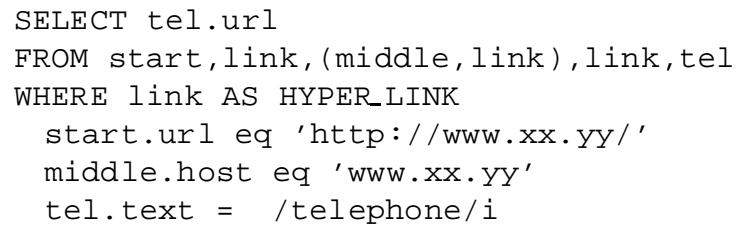

Figure 7. A query example with a self-loop.

The starting URL is given on line 4. The from-clause on line 2 denotes the search path expression that includes the self-loop (middle, link). The self-loop expresses a non-deterministic search path. The link is a hyperlink as denoted on line 3 and the condition of middle is stated on line 5 to restrict the search to HPs stored in the server host ' www. $\mathrm{xx} \cdot \mathrm{yy}^{\prime}$. The condition on line 6 matches if the HP tel includes the string telephone. The search result is output using the format on line 1.

The self-loop enables a search of all HPs that include the keyword telephone even if the hyperlink structure of the Web site ' www $x x \cdot y y^{\prime}$ is semi-structured.

(3) Example in Section 1

In the scenario in Section 1, the search example is divided into two queries. The first query is "search for a post office that is nearest to my university and is also open on Saturday." The second query is "search for Italian restaurants within 1 kilometer of the selected post office that are mentioned on a restaurant information Web site and have a long wine list."

In the first query, we use the condition "within 500 meters" instead of "nearest to" because the latter is not suitable. The starting HP is the HP of John's university and the target is the post office's HP that is within 500 meters of the university and open on Saturday. This query is shown in Fig. 8.

SELECT schedule.url

2 FROM university, distance link, (postoffice, link), link, schedule

3 WHERE link AS HYPERLINK

4 university.url eq 'http://www.zz.edu/'

5 distance_link

AS Distance (university, postoffice) < 500 postoffice.title $=$ /post office/i schedule.text $=/$ open on saturday/i

Figure 8. The first query of the scenario.

The starting URL of the university's HP is given on 
line 4. The search path is from university to schedule as mentioned in the from-clause on line 2. This path is the sequence of conditions described above and includes the selfloop (postoffice, link). The self-loop and its conditions restrict the search range to the inside of the post office's Web site. The link on line 3 denotes a hyperlink and the distance_link on line 5 is defined as a generic link created by evaluating the distance function Distance between the objects mapped from the HPs. The conditions on lines 6 and 7 match if the HP attributes include each specified keyword. The schedule.url on line 1 specifies the result format.

The second query starts from an information site of restaurants. The search proceeds to the Italian restaurants that are listed on the site, are located within 1 kilometer of the post office, and include the keyword "wine." The search expression is described in Fig.9.

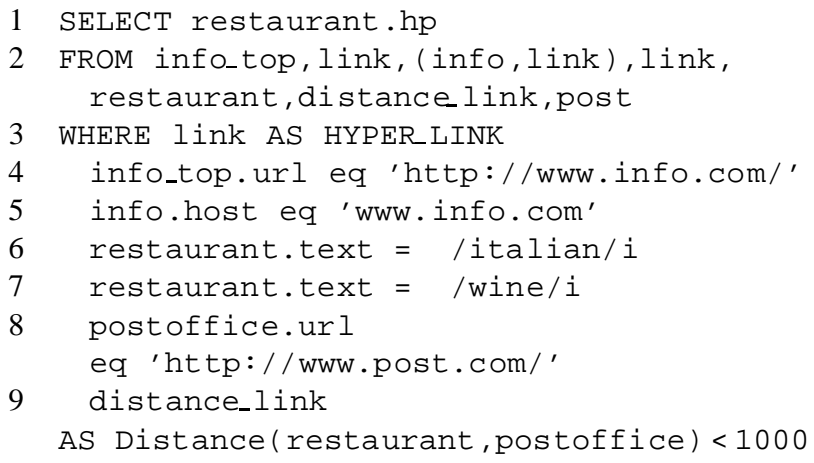

Figure 9. The second query of the scenario.

The starting URL of the HP of the info_top is given on line 4. The search path from the HP of the info_top to the post office's HP denoted on line 2 is also the sequence of conditions described in the where-clause and includes the self-loop (info,link). The host name info.host on line 5 is the server host that the middle HPs on the search path are stored in. The target rest aurant's HP appears in the middle of the path so that it is connected to the post office by a generic link. The post of fice's last HP on line 8 is a static URL given by the first query and the distance_link on line 9 between the restaurant and the postoffice, is the generic link that is produced if the distance is under 1 kilometer. The conditions on lines 6 and 7 match if the restaurant. text includes both strings.

\section{Augmented Web Space in Digital City Kyoto}

We are providing a 3D walk-through implementation and a map-based interface as free services for Digital City Kyoto $^{3}$. These services are based on our idea of augmented

\footnotetext{
${ }^{3}$ http://www.digitalcity.gr.jp/
}

Web spaces and their query language.

\subsection{System Architecture}

Figure 10 shows a system architecture including services of Digital City Kyoto. The system consists of a query processor, a search controller, a Web module, a generic link module with a GIS, a cache module, a generic link database, and a formatter.

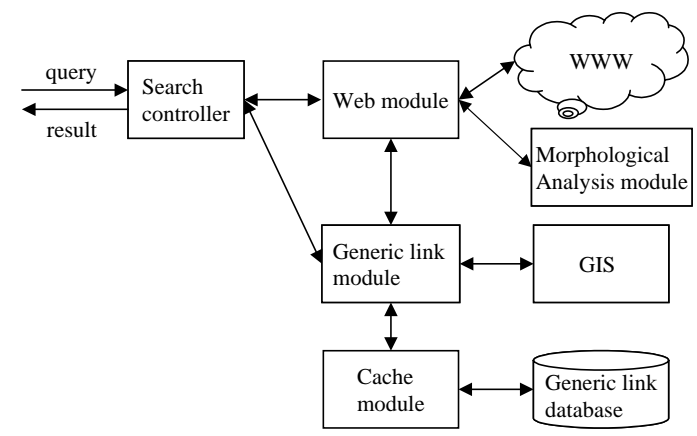

Figure 10. System architecture.

The system works as follows. The query processor receives a search query from an interface, extracts conditions for HPs and links in the where-clause from the query, binds the conditions to members of a search path expression in the from-clause, and carries out transfer to the search controller. The search controller extracts HPs and links designated in the search path based on typical search algorithms such as depth-first search and breadth-first search in cooperation with a Web module and a generic link module. The formatter handles each search result from the search module and outputs result attributes denoted in the select-clause.

In accordance with the search controller, the Web module retrieves HPs from the Internet. The Web module parses the retrieved HPs into HP attributes with an HTML parser and a morphological analyzer, and then evaluates the designated conditions and extracts hyperlinks in the HPs. The generic link module creates generic links that cooperate with the Web module and a GIS. To begin with this creation, the generic link module utilizes the Web module for retrieving geographical attributes of a source HP of a generic link. Next, the generic link module applies the geographical attributes and relational conditions to the GIS. The GIS identifies coordinates of the source HP on the geographical map by its attributes and searches for targets that match the geographical relational conditions from the source. Finally, the generic link module searches for HPs corresponding to the targets and outputs pairs of the source HP and a target HP as generic links. The generic links are created as mentioned above; however, sequential generation of the generic links 
takes several seconds because the generation requires the repetition of time-consuming processes such as accessing HPs on the Internet and searching for objects in the GIS. To provide search services for a great number of users in Digital City Kyoto, it is important to reduce the response time of the services. Accordingly, we have integrated a cache module of generic links that are based on more than 2600 HPs with precise $\mathrm{XY}$ coordinates into the generic link module.

\subsection{Interfaces in Digital City Kyoto}

\section{(1) 3D Walk-through}

A 3D walk-through implementation of Shijo street in Kyoto city (Fig.12) is available in Digital City Kyoto. The walk-through is described in 3DML [1], which is a simple modeling language for creating 3D spaces composed of 3D bitmaps like building blocks. We can browse the walkthrough on major Web browsers with the 3DML plug-in provided by Flatland, Inc [1]. In this walk-through, each tile on the road has a link to a CGI script that searches for HPs close to where the agent (i.e., user's position) stands. The expression in Fig. 11 denotes a query call by the CGI script.

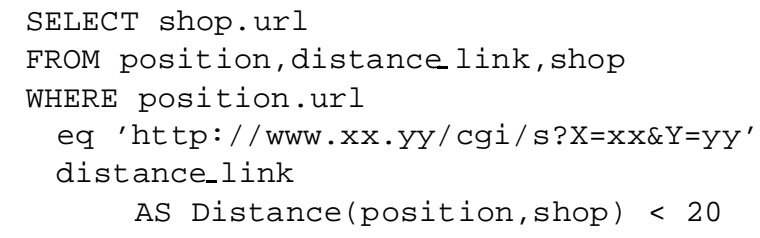

Figure 11. A query used in a 3D walk-through.

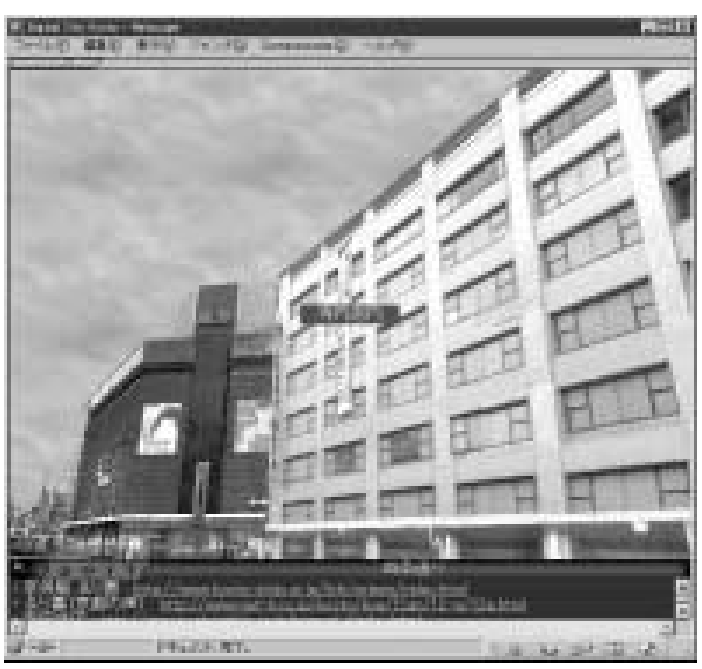

Figure 12. 3D walk-through.
This query means that the starting HP of the place where the agent stands is set by the CGI script on line 3. Therefore, we can evaluate the distances between this position and HPs in the database by using the generic link Distance on Line 4 and outputting the HPs within 20 meters according to the format on line 1 .

\section{(2) Map-based Interface}

A map-based interface (Fig.13) is one of our service HPs for Digital City Kyoto. The Java applet of the digitized map shows a search result for an inquiry based on the augmented Web space and its query language. HPs in the search result are displayed as icons for users to jump to them.

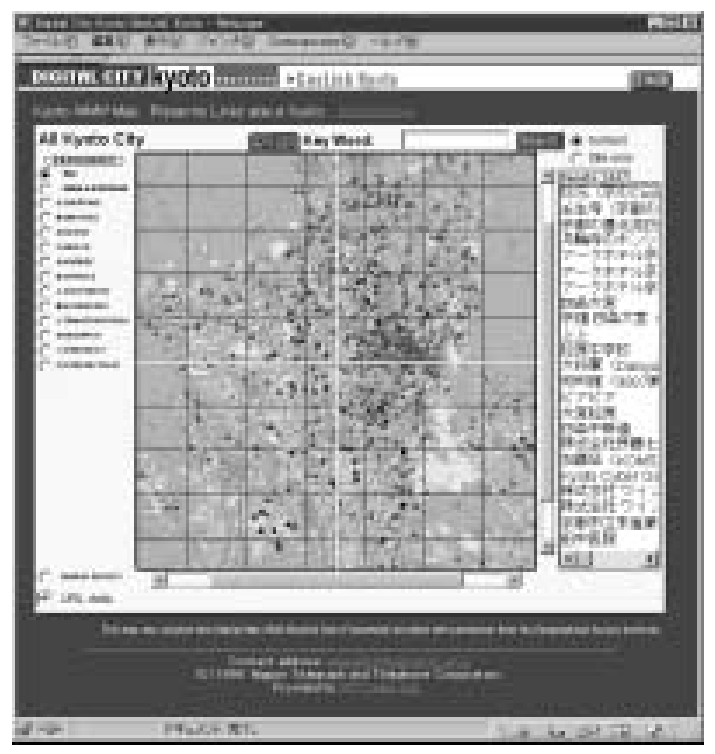

Figure 13. Map-based interface.

This version of Digital City Kyoto based on augmented Web spaces is partially open on the Internet and search in these spaces is available on the grounds of the query language. The map-based interface enables users to search for targets by distance, keywords, and some HP categories. The map-based interface also allows the tracing of a search path with conditions about geographical properties and HP attributes from a specified HP.

\subsection{Towards Interactive Search Interface}

Queries for current interfaces in Digital City Kyoto are not very complicated because users always break down their requests into simple operations that are acceptable by these interfaces. To describe a search expression that includes a user's vague search intention without breaking it down, it is necessary to extend functions of the map-based interface and to utilize interface agents.

(1) Expansion of Map-based Interface 
The map-based interface has the advantage of displaying a lot of data simultaneously. However, it is hard to coordinate a search path that consists of several sub goals in an intuitive interface operation. For example, if a user wants to search for a nearby station from a building that is a previous search result, the user has to mark the building and search related targets on condition of keywords and geographical relationships using some other separated windows or detached menus. To avoid these complicated operations, it is important to develop intuitive interface systems able to integrate natural language processing to grasp a user's vague intention and operational indicators based on the user's operation history.

\section{(2) Utilization of Interface Agents}

As described in [8], an interface agent can actively assist a user in operating an interface. The user can discuss his/her requests with the interface agent and can obtain search results or recommendations. The interface agent can convert a whole request to a single query to make full use of the query language to generate a search expression. The interface agent can also create complex queries by incorporating background knowledge and the user's history.

\section{Conclusion}

We have proposed an augmented Web space that integrates a GIS and the WWW to support everyday life in a metropolitan area. We have also described a query language for the augmented Web space, which consists of search path expressions and geographical evaluation functions. The search path expressions are used to navigate semi-structured data such as augmented Web spaces. The geographical evaluation functions are used to dynamically create generic links based on geographical relations between HPs.

We are now working on query optimization to search the augmented Web spaces efficiently. In an actual search, searching for the target directly according to the search path expression is not the best way. There are better possibilities such as swapping the evaluation order depending on the size of the data set. We are also considering an interface that enables us to query interactively by using natural language processing.

\section{References}

[1] Flatland, inc. http://www.flatland.com/.

[2] S. Abiteboul. Querying Semi-Structured Data. In Database Theory - ICDT '97, 6th International Conference, pages 118, 1997.

[3] S. Chakrabarti, D. Gibson, and K. McCurley. Surfing the Web backwards. In Proceedings of 8th International World Wide Web Conference, 1999.
[4] S. Green. Automated link generation: can we do better than term repetition? In Proceedings of 7th International World Wide Web Conference, 1998.

[5] T. Ishida, J. Akahani, K. Hiramatsu, K. Isbister, S. Lisowski, H. Nakanishi, M. Okamoto, Y. Miyazaki, and K. Tsutsuguchi. Digital City Kyoto: Towards A Social Information Infrastructure. In Cooperative Information Agents III, pages 34-46, 1999.

[6] D. Konopnicki and O. Shmueli. W3QS: A Query System for the World-Wide Web. In Proceedings of the 21st International Conference on Very Large Data Bases, pages 54-65, 1995.

[7] D. Konopnicki and O. Shmueli. Information Gathering in the World Wide Web: The W3QL Query Language and the W3QS System. ACM Transactions on Database Systems, 23(4):369-410, 1998.

[8] H. Lieberman. Autonomous Interface Agent. In Proceedings of the ACM Conference on Computer and Human Interface, CHI-97, pages 67-74, 1997.

[9] A. Mendelzon, G. Mihaila, and T. Milo. Querying the World Wide Web. International Journal on Digital Libraries, 1(1):54-67, 1997.

[10] A. Mendelzon and T. Milo. Formal Models of Web Queries. In Proceedings of the 16th ACM Symposium on Principles of Database Systems, pages 134-143, 1997.

[11] X. Meng, R. Fowler, and E. Rieken. Bridging the Gap Between GIS and the WWW. In Proceedings of 6th International World Wide Web Conference, 1997.

[12] I. Open GIS Consortium. OpenGIS Simple Features Specification For SQL Revision 1.0. http://www.opengis.org/.

[13] M. Potmesil. Map Alive: Viewing Geospatial Information on the WWW. In Proceedings of 6th International World Wide Web Conference, 1997.

[14] Q. Qian, M. Tanizaki, and K. Tanaka. Abstraction and Inheritance of Hyperlinks in an Object-Oriented Hypertext Database System TextLink/Gem. IEICE Transactions on Information and Systems, E78-D(11):1343-1353, 1998.

[15] K. Tanaka, N. Nishikawa, S. Hirayama, and K. Nanba. Query Pairs As Hypertext Links. In Proceedings of 7th IEEE Data Engineering Conference, pages 456-463, 1991. 